\title{
Successful low-dose azathioprine for myasthenia gravis despite hepatopathy from primary sclerosing cholangitis: a case report
}

\author{
Josef Finsterer, Sonja Höflich
}

\begin{abstract}
Introduction: Although myasthenia gravis is frequently associated with other disorders, it has not been reported together with primary sclerosing cholangitis, complicating the administration of liver-toxic immunosuppressive therapy.

Case presentation: A 73-year-old Caucasian woman with a history of arterial hypertension, thyroid dysfunction, glaucoma, right-sided ptosis and later generalized weakness, was diagnosed with myasthenia gravis. Additionally, primary sclerosing cholangitis was detected, initially prohibiting the administration of immunosuppressants. Despite treatment with steroids and pyridostigmine she repeatedly experienced myasthenic crises. After the fifth crisis and after antibody titers had reached levels $>100 \mathrm{nmol} / \mathrm{L}$ during two years of follow-up, it was decided to restart azathioprine. Interestingly, low-dose azathioprine $(1.5 \mathrm{mg} / \mathrm{kg} /$ day $)$ was well tolerated, had a positive clinical and immunological effect and did not worsen primary sclerosing cholangitis.

Conclusion: Myasthenia gravis may occur together with primary sclerosing cholangitis in the same patient. Mild immunosuppression with azathioprine is feasible and effective in such a patient, without worsening myasthenia gravis or primary sclerosing cholangitis.
\end{abstract}

\section{Introduction}

Myasthenia gravis (MG) may be due to a genetic defect, intoxication, or most frequently, autoimmune mechanisms [1]. Although autoimmune MG is frequently associated with other autoimmune disorders [2-13], to the best of our knowledge the association of MG with primary sclerosing cholangitis (PSC) has not been reported.

\section{Case presentation}

Our patient is a 73-year-old Caucasian woman with a history of elevated liver function parameters since age 71 and isolated right-sided ptosis one month later. Within two weeks she also developed easy fatigability, weakness when climbing stairs, ophthalmoparesis, and dysphagia for solid food, prompting hospitalization. Diagnostic work-up revealed elevated antibodies against postsynaptic acetylcholine-receptors (AchR-ab) with a titer of $35.7 \mathrm{nmol} / \mathrm{L}$ (normal $<0.4 \mathrm{nmol} / \mathrm{L}$ ) (figure 1),

\footnotetext{
* Correspondence: fipaps@yahoo.de

Krankenanstalt Rudolfstiftung, Juchg. 25, 1030, Vienna, Austria
}

repetitive nerve stimulation indicative of a postsynaptic transmission defect, and a positive tensilon test, so pyridostigmine and prednisolone $(25 \mathrm{mg} /$ day $)$ were started (figure 2). Further diagnostic work-up revealed a mediastinal tumor and elevated liver function parameters (figure 3).

On hospital day 11 she experienced a myasthenic crisis, requiring intubation, intravenous administration of neostigmine and immunoglobulins, and plasmapheresis six times. Resection of the mediastinal tumor on hospital day 13 revealed a thymoma B3, without indication for chemotherapy. After surgery pyridostigmine was restarted but had to be replaced by neostigmine on hospital day 34 due to better efficacy. Azathioprine was initiated on hospital day 34 in a reduced dosage $(50 \mathrm{mg} /$ day) because of hepatopathy and increased to $100 \mathrm{mg} /$ day on hospital day 44 (figure 2). On hospital day 47 she experienced a second myasthenic crisis and again required intensive care. On hospital day 57 a third myasthenic crisis manifested with dysphagia, dyspnea, and respiratory failure, again requiring intensive care. On hospital day 72, azathioprine was

\section{C) Biomed Central}




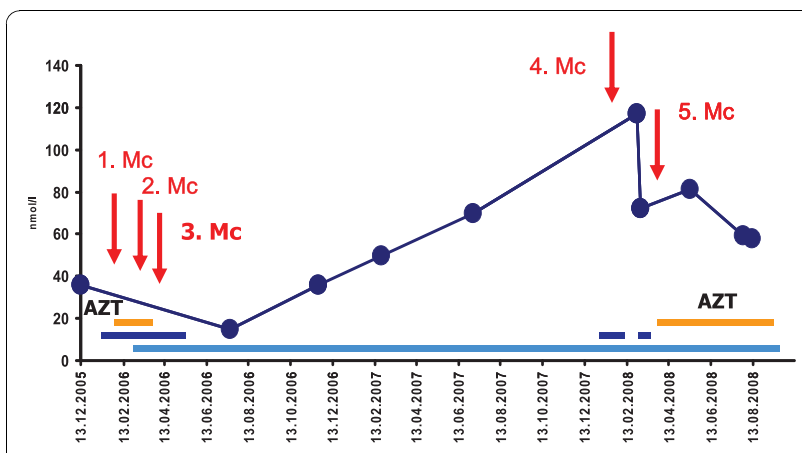

Figure 1 Course of AchR-ab over almost three years, showing a positive effect of immunosuppression at onset and after the fifth myasthenic crises despite PSC.

increased to $150 \mathrm{mg} /$ day while prednisolone remained at $25 \mathrm{mg} /$ day (figure 2). Upon diagnostic work-up for further increase of liver function parameters, magnetic resonance (MR)-cholangiography revealed PSC with negative antinuclear antibodies (ANA), smooth-muscle antibodies, anti-mitochondrial antibodies, liver-kidney antibodies, or soluble liver antigen. Ursodesoxycholic acid was given and azathioprine was discontinued (figure 2). At discharge on hospital day 108 she was under prednisolone (15 mg/day), pyridostigmine (360 mg/day), glimepiride (7 mg/day) for mild diabetes, ursodesoxycholic acid (1250 mg/day), calcium, and alendrone (70 mg once a week) for osteoporosis (figure 2). Except for right-sided ptosis, she was symptomfree.

Two months after dismissal AchR-ab reached its lowest level (figure 1) so steroids were reduced to $10 \mathrm{mg} /$ day. At age 72 years, prednisolone was further reduced to $5 \mathrm{mg}$ / day. Six months later she presented with right-sided ptosis, slight weakness, wasting of the thighs, exaggerated patella tendon reflexes and reduced Achilles tendon reflexes. Pyridostigmine was increased to $480 \mathrm{mg} /$ day and prednisolone reduced to $2.5 \mathrm{mg} /$ day. Three months

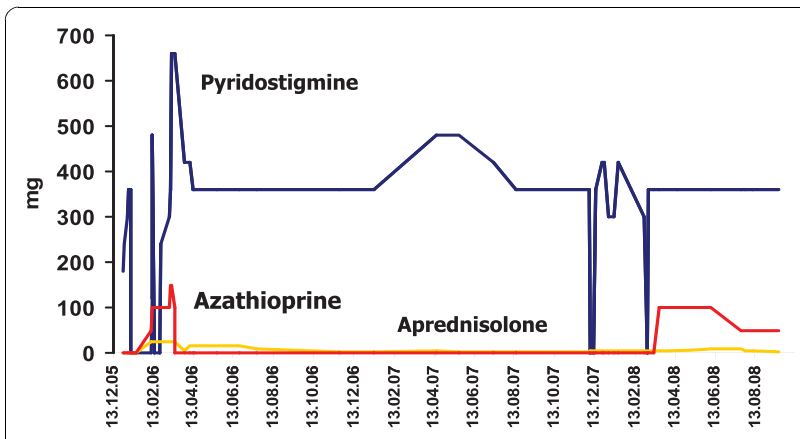

Figure 2 Courses of pyridostigmine, prednisolone, and azathioprine dosages over a follow-up period of almost three years, showing that corticosteroids were given throughout this period in low dosages and that pyridostigmine dosages varied widely because of the clinical instability. later pyridostigmine was reduced to $360 \mathrm{mg} /$ day without a relapse. At age 73 years she experienced a fourth myasthenic crisis during an infectious disease, requiring intubation and mechanical ventilation. After increase of prednisolone and pyridostigmine she made a full recovery. A fifth myasthenic crisis occurred five months later, which responded simply to switching from pyridostigmine to neostigmine intravenously. At that time it was decided to restart azathioprine in a dosage of $100 \mathrm{mg} /$ day because of recurrent myasthenic crises and maximal elevation of AchR-ab to $117.03 \mathrm{nmol} / \mathrm{L}$ (figures 1 and 2). Because of azathioprine-induced elevation of liver function parameters (figure 3) azathioprine had to be reduced to $50 \mathrm{mg} /$ day. At age 74 years corticosteroids were discontinued and azathioprine increased to $75 \mathrm{mg} /$ day and later $87.5 \mathrm{mg} /$ day, without further elevation of liver function parameters. Under this regimen MG did not recur and AchR-ab levels remained low until the last follow-up at age 75 years.

\section{Discussion}

Our patient is not only interesting for the association of MG with PSC, but also for resection of a thymoma two days after onset of the first myasthenic crisis, for the transient administration of drugs, such as calcium, known to increase the risk for myasthenic crises, for the continuous rise of the AchR-ab under steroids, and for the effectiveness and tolerance of azathioprine despite its liver toxicity. Autoimmune MG is frequently associated with other autoimmune disease, including primary biliary cirrhosis (table 1), but has not been reported together with PSC as in the presented case. PSC is frequently associated with autoimmune disorders [14], such as pancreatitis, colitis ulcerosa, or Crohn's disease, of which none was found in our patient, and responds favorably to azathioprine [15]. Concerning the optimal timing of thymectomy there is general consensus that it should be

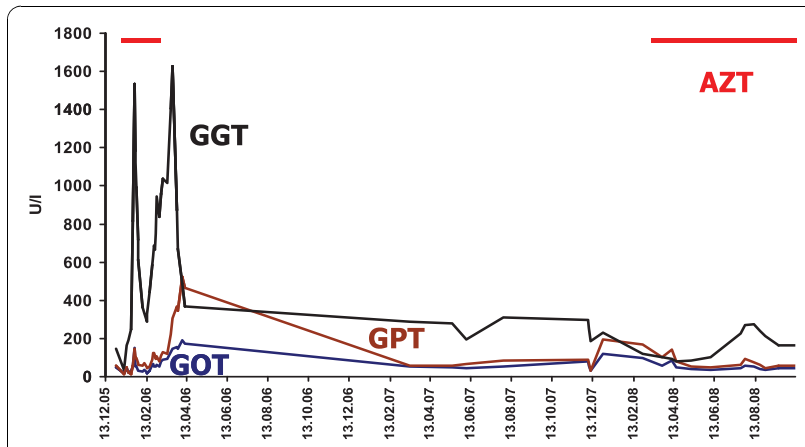

Figure 3 Course of GOT (glutamic oxaloacetic transaminase), GPT (glutamate pyruvate transaminase) and GGT (gammaglutamyl transpeptidase) over almost three years in a patient with MG and PSC. GGT reached excessively high levels, particularly at onset of the disease, where three myasthenic crises occurred. 
Table 1 Autoimmune disorders frequently associated with MG

\begin{tabular}{ll}
\hline Disorder & Reference \\
\hline Lupus erythematosus & {$[9]$} \\
\hline Polymyositis & {$[4]$} \\
\hline Rheumatoid arthritis & {$[12]$} \\
\hline Graves' disease & {$[3]$} \\
\hline Diabetes mellitus type 1 & {$[13]$} \\
\hline Hashimoto's thyroiditis & {$[3]$} \\
\hline Scleroderma & {$[5]$} \\
\hline Alopecia areata & {$[7]$} \\
\hline Giant cell myocarditis & {$[6]$} \\
\hline Primary biliary cirrhosis & {$[8]$} \\
\hline Bronchial asthma & {$[10]$} \\
\hline Addison's disease & {$[11]$} \\
\hline Autoimmune pancreatitis & {$[2]$} \\
\hline
\end{tabular}

carried out as soon as possible. Whether thymectomy during a myasthenic crisis may jeopardize the patient, or may prolong hospitalization and should only be carried out after pre-operative stabilization is under debate. However, there are indications that thymectomy can be safely performed even in patients with uncontrolled MG if there is proper pre-operative preparation, good anesthetic management, and optimal peri-operative respiratory care [16].

Despite the known intolerance and contraindications for azathioprine in PSC, it was decided to restart azathioprine because it was regarded to be effective and to have the lowest rate of side-effects among all immunosuppressants used for MG (table 2), because levels of liver function parameters were in a tolerable range, because our patient needed immunosuppressive therapy, and because the intolerability to azathioprine at age 71 years occurred during a myasthenic crisis and after surgery. Since our patient did not tolerate $100 \mathrm{mg} /$ day of azathioprine, the dosage was first reduced to $50 \mathrm{mg} /$ day and later to $87.5 \mathrm{mg} /$ day, dosages under which AchR-ab continuously declined and no further myasthenic crises occurred during the next two

Table 2 Treatment options for primary sclerosing cholangitis.

\begin{tabular}{ll}
\hline Agent & Reference \\
\hline Ursodeoxycholic acid & {$[17-19]$} \\
\hline Prednisolone (initially $1 \mathrm{mg} / \mathrm{kg} /$ day) & {$[17-20]$} \\
\hline Azathioprin $(1-2.5 \mathrm{mg} / \mathrm{kg} /$ day) & {$[[17]$, present case, [17-21]] } \\
\hline Methotrexate & {$[22]$} \\
\hline Tacrolimus & {$[18]$} \\
\hline Endoscopic dilation of bile duct stricutres & {$[23]$} \\
\hline Liver transplantation & {$[18,24]$} \\
\hline
\end{tabular}

years. Steroids in a low dosage were the mainstay of therapy during three years but were discontinued because they were ineffective at reducing the high levels of AchR-ab and were associated with side-effects, such as diabetes and osteoporosis. Discontinuation of steroids, which may have a favorable effect on PSC in single patients, did not worsen PSC. Why, contrary to other MG manifestations, rightsided ptosis hardly resolved, remains speculative. It is possible that she had another disorder in addition to MG, such as a multi-system metabolic disease. Overall, management of MG becomes a challenge if the patient is unstable, if AchR-ab continuously increases, and if there are contraindications for immunotherapy. However, when closely monitoring a patient for myasthenic symptoms and liver disease, it is even possible to give a liver toxic drug instead of more costly immunoglobulins or repeated plasmaphereses. The outcome may be further improved if potentially dangerous drugs in MG are avoided and close monitoring and regular re-evaluation of the medication for potential contraindications is carried out.

\section{Conclusions}

MG may occur together with PSC in the same patient. Immunosuppression with azathioprine in PSC and MG with progressively increasing high antibody titers is feasible, safe, and effective, even with reduced dosages, provided there is close monitoring of AchR-ab and liver function parameters.

\section{Consent}

Written informed consent was obtained from the patient for publication of this case report and any accompanying images. A copy of the written consent is available for review by the Editor-in-Chief of this journal.

\section{Authors' contributions}

JF analyzed and interpreted the patient data regarding the blood chemical, immunological and electrophysiological investigations. SH performed some of the clinical examination and was a contributor in writing the manuscript. All authors read and approved the final manuscript.

\section{Competing interests}

The authors declare that they have no competing interests.

Received: 24 January 2010 Accepted: 8 November 2010 Published: 8 November 2010

\section{References}

1. Vincent A, Bowen J, Newsom-Davis J, McConville J: Seronegative generalised myasthenia gravis: clinical features, antibodies, and their targets. Lancet Neurol 2003, 2:99-106.

2. Colaut F, Toniolo L, Sperti C, Pozzobon M, Scapinello A, Sartori CA: Autoimmune-like pancreatitis in thymoma with myasthenia gravis. Chir Ital 2002, 54:91-94.

3. Kanazawa M, Shimohata T, Tanaka K, Nishizawa M: Clinical features of patients with myasthenia gravis associated with autoimmune diseases. Eur J Neurol 2007, 14:1403-1404.

4. Inoue M, Kojima Y, Shinde A, Satoi H, Makino F, Kanda M, Shibasaki H: Concurrence of myasthenia gravis, polymyositis, thyroiditis and 
eosinophilia in a patient with type B1 thymoma. Rinsho Shinkeigaku 2007, 47:423-428.

5. Zivković SA, Medsger TA Jr: Myasthenia gravis and scleroderma: two cases and a review of the literature. Clin Neurol Neurosurg 2007, 109:388-391.

6. Kanzato N, Nakasone I, Sunagawa O, Komine Y, Fukiyama K: Giant-cell myocarditis without a symptom of heart failure seen in a patient with myasthenia gravis and concurrent Hashimoto's disease. Rinsho Shinkeigaku 2001, 41:813-817.

7. Suzuki S, Shimoda M, Kawamura M, Sato H, Nogawa S, Tanaka K, Suzuki N, Kuwana M: Myasthenia gravis accompanied by alopecia areata: clinical and immunogenetic aspects. Eur J Neurol 2005, 12:566-570.

8. Horigome H, Nomura T, Saso K, Joh T, Ohara H, Akita S, Sobue S, Mizuno Y, Kato Y, Itoh M: Coexistence of primary biliary cirrhosis and myasthenia gravis: a case study. Hepatogastroenterology 2000, 47:125-127.

9. Aracena CR, Nogales-Gaete J: Coexistence of myasthenia gravis and systemic lupus erythematosus: Is it more frequent than we thought? Rev Med Chil 2006, 134:1203-1205.

10. Souza-Machado A, Ponte E, Cruz AA: Severe asthma associated with myasthenia gravis and upper airway obstruction. J Investig Allergol Clin Immunol 2007, 17:267-270.

11. Okada T, Kawamura T, Tamura T, Toshima R, Sakai O: Myasthenia gravis associated with Addison's disease. Intern Med 1994, 33:686-688.

12. Kerkeni $\mathrm{S}$, Marotte $H$, Miossec $\mathrm{P}$ : Improvement with rituximab in a patient with both rheumatoid arthritis and myasthenia gravis. Muscle Nerve 2008, 38:1343-1345.

13. Toth C, McDonald D, Oger J, Brownell K: Acetylcholine receptor antibodies in myasthenia gravis are associated with greater risk of diabetes and thyroid disease. Acta Neurol Scand 2006, 114:124-132.

14. Shorbagi A, Bayraktar Y: Primary sclerosing cholangitis - what is the difference between east and west? World I Gastroenterol 2008, 14:3974-3981

15. Saich R, Chapman R: Primary sclerosing cholangitis, autoimmune hepatitis and overlap syndromes in inflammatory bowel disease. World J Gastroenterol 2008, 14:331-337.

16. Datt V, Tempe DK, Singh B, Tomar AS, Baneriee A, Dutta D, Bhandari H: Anesthetic management of patient with myasthenia gravis and uncontrolled hyperthyroidism for thymectomy. Ann Card Anaesth 2010, 13:49-52.

17. Mueller T, Bianchi L, Menges M: Autoimmune hepatitis 2 years after the diagnosis of primary sclerosing cholangitis: an unusual overlap syndrome in a 17-year-old adolescent. Eur J Gastroenterol Hepatol 2008, 20:232-236.

18. Oya H, Sato Y, Yamamoto S, Takeishi T, Kobayashi T, Hatakeyama K: Living related donor liver transplantation for primary sclerosing cholangitis with hepatocellular carcinoma and Crohn's disease: a case report. Transplant Proc 2004, 36:2297-2298.

19. Takiguchi J, Ohira H, Rai T, Shishido S, Tojo J, Sato Y, Kasukawa R, Watanabe H, Funabashi Y, Kumakawa H: Autoimmune hepatitis overlapping with primary sclerosing cholangitis. Intern Med 2002, 41:696-700.

20. Burak KW, Angulo P, Lindor KD: Is there a role for liver biopsy in primary sclerosing cholangitis? Am J Gastroenterol 2003, 98:1155-1158.

21. CadahÃa V, Rodrigo L, Fuentes D, Riestra S, de Francisco R, FernÃindez M: Celiac disease (CD), ulcerative colitis (UC), and primary sclerosing cholangitis (PSC) in one patient: a family study. Rev Esp Enferm Dig 2005, 97:907-913.

22. Lee YM, Kaplan MM: Treatment of primary biliary cirrhosis and primary sclerosing cholangitis: use of ursodeoxycholic acid. Curr Gastroenterol Rep 1999, 1:38-41.

23. Holtmeier J, Leuschner U: Medical treatment of primary biliary cirrhosis and primary sclerosing cholangitis. Digestion 2001, 64:137-150.

24. BroomÃ@ U, Glaumann H, Lindstöm E, Lööf L, Almer S, Prytz H, SandbergGertzÃ@n H, Lindgren S, Fork FT, JÃarnerot G, Olsson R: Natural history and outcome in 32 Swedish patients with small duct primary sclerosing cholangitis (PSC). J Hepatol 2002, 36:586-589.

doi:10.1186/1752-1947-4-356

Cite this article as: Finsterer and Höflich: Successful low-dose azathioprine for myasthenia gravis despite hepatopathy from primary sclerosing cholangitis: a case report. Journal of Medical Case Reports 2010 4:356.

\section{Submit your next manuscript to BioMed Central and take full advantage of:}

- Convenient online submission

- Thorough peer review

- No space constraints or color figure charges

- Immediate publication on acceptance

- Inclusion in PubMed, CAS, Scopus and Google Scholar

- Research which is freely available for redistribution 